\title{
El consejo pastoral como práctica de sabiduría
}

\author{
O conselho pastoral como prática da sabedoria
}

\author{
Pastoral counseling as wisdom practice
}

\section{Daniel Schipani}

Ph.D. com concentração em Teologia Prática pelo Princeton Theological Seminary, Doutor em Psicologia pela Universidade Católica Argentina, Master of Arts: Peace Studies, por Goshen Biblical Seminary, ministro ordenado da Igreja Menonita, EUA, professor de Cuidado e Conselho Pastoral no Associated Mennonite Biblical Seminary, Elkhart, IN - EUA, e-mail: dschipani@ambs.edu

\section{Resumen}

Este ensayo desarrolla la tesis principal que inspira mi trabajo práctico y teórico en torno al consejo pastoral: se trata de una forma especial de ministerio cristiano centrado en la sabiduría espiritual más bien que en la salud mental como su metáfora básica. La preocupación principal es cómo acompañar a personas a que vivan mejor, más sabiamente; esto es lo que quiero decir con la meta general de ayudarles a despertar, ejercitar, y desarrollar su inteligencia moral y espiritual en medio de los desafíos y luchas que les presenta la vida cotidiana.

Palabras-clave: Consejo pastoral. Sabiduría espiritual. Salud mental. Inteligencia espiritual. 


\section{Resumo}

Este ensaio desenvolve a tese principal que inspira meu trabalho prático e teórico em torno do Aconselhamento Pastoral: trata-se de uma forma especial de ministério cristão focado mais na sabedoria espiritual do que na saúde mental como sua metáfora de base. A preocupação principal é como acompanhar pessoas para que vivam melhor, com mais sabedoria - é isto que quero dizer com o objetivo geral de ajudar a despertar, exercitar e desenvolver sua inteligência moral e espiritual em meio aos desafios e lutas que a vida cotidiana Ihes apresenta.

Palavras-chave: Aconselhamento Pastoral. Sabedoria espiritual. Saúde mental. Inteligência espiritual.

\section{Abstract}

This essay develops the thesis that inspires my practical and theoretical work in pastoral counseling: pastoral counseling is a special form of Christian ministry centered in spiritual wisdom (rather than mental health) as its core metaphor. The counselor's main concern is how to accompany people facing life challenges and struggles so that they can live better, wiser lives. This is what I mean by claiming that the overarching goal is to help them to awaken, practice, and develop their spiritual intelligence.

Keywords: Pastoral counseling. Spiritual wisdom. Mental health. Spiritual intelligence.

\section{Introducción}

Este ensayo desarrolla la tesis principal que inspira mi trabajo práctico y teórico en torno al consejo pastoral: ${ }^{1}$ se trata de una forma especial de ministerio cristiano centrado en la sabiduría espiritual más bien que en la salud mental como su metáfora básica. Obviamente, todo lo que hacemos en consejo pastoral depende en parte de la situación de salud

1 Para una presentación detallada y sistemática de mi modelo de consejo pastoral, véase Daniel S. Schipani, 0 caminho da sabedoria no aconselhamento pastoral, Sinodal, S. Leopoldo, 2004; Daniel S. Schipani, ed., Mennonite Perspectives on Pastoral Counseling, IMS, Elkhart, 2007, cap. 1 y 9; y El consejo pastoral como camino de sabiduría, Ediciones Kairós, Buenos Aires, en preparación.

Rev. Pistis Prax., Teol. Pastor., Curitiba, v. 3, n. 2, p. 405-423, jul./dez. 2011 
mental de las personas involucradas y tiende a su vez a afectar la condición de salud mental de diversas maneras. Sin embargo, la preocupación principal es otra: cómo acompañar a tales personas a que vivan mejor, más sabiamente; esto es lo que quiero decir con la meta general de ayudarles a despertar, ejercitar, y desarrollar su inteligencia moral y espiritual en medio de los desafíos y luchas que les presenta la vida cotidiana.

Necesitamos seguir privilegiando las perspectivas y contribuciones teológico-pastorales y éticas, incluyendo criterios de "vida buena" y plenitud humana”, porque en el consejo pastoral tienen prioridad sobre las perspectivas y contribuciones provenientes de la psicología y la psiquiatría, aunque estas últimas son indispensables. En otras palabras, en términos epistemológicos como también metodológicos, afirmamos que la teología y las ciencias humanas son ambas esenciales y deben considerarse juntas pero sin reducir la una a la otra; pero afirmamos también que la relación interdisciplinaria no es simétrica sino que la teología tiene prioridad conceptual sobre la psicología (HUNSINGER, 1995; SCHIPANI; JIMÉNEZ, 1997b). ${ }^{2}$

Según este nuevo modelo, en el consejo pastoral procuramos promover el emerger humano mediante una forma de acompañamiento de personas, parejas, familias, o pequeños grupos quienes enfrentan desafíos y luchas existenciales. La expresión emerger humano en este contexto denota un proceso de humanización entendido principalmente en perspectiva teológica. Se trata de llegar a ser "más humanos" en términos de (nuestra comprensión de) la dádiva y la promesa divina de libertad y plenitud humana auténticas. Además, la frase connota el desarrollo humano según el marco ético-escatológico más amplio simbolizado bíblicamente como el Reino de Dios, es decir, la comunidad normativa de amor, justicia y paz. Por lo tanto, el proceso de "emerger" involucra aquella formación y transformación en la vida de personas, familias, y comunidades que podemos asociar con el crecimiento moral y espiritual de naturaleza

2 Sobre este punto véase Deborah van Deusen Hunsinger, Theology \& Pastoral Counseling: A New Interdisciplinary Approach, Eerdmans, Grand Rapids, 1995. Hunsinger presenta sistemáticamente una propuesta fundamentada en el pensamiento de Karl Barth. Véase también Daniel S. Schipani, "Bases eclesiológicas: la iglesia como comunidad sanadora”, en Daniel S. Schipani y Pablo A. Jiménez, eds. Psicología y consejo pastoral: perspectivas hispanas, AETH, Decatur, 1997a, p. 3-26. 
cristomórfica. Se desprende entonces que, por un lado, esta forma de entender el emerger humano no debe identificarse meramente con las nociones psicológicas de desarrollo y maduración; por otro lado, nuestra práctica y teoría del consejo pastoral debe establecer ciertas conexiones con el desarrollo humano "natural" y con las perspectivas psicológicas sobre el desarrollo humano.

Reitero por lo tanto que el propósito general del consejo pastoral, expresado en sencillas palabras, es ayudar a las personas, parejas, familias y otros, a que puedan vivir más sabiamente a la luz de Dios. Nuestra práctica se encamina fundamental pero no exclusivamente, a estimular, nutrir, y desarrollar la inteligencia moral y espiritual de las personas aconsejadas. Podemos decir que la inteligencia moral y espiritual consiste en saber cómo vivir bien, especialmente en medio de situaciones conflictivas, crisis, sufrimiento, pérdida, y la misma muerte. ${ }^{3}$ Por lo tanto, este ministerio puede verse como una dimensión de la labor más amplia del cuidado cuya meta es la salud y el bienestar integral.

En los párrafos que siguen presento tal comprensión del consejo pastoral como ministerio de la iglesia e ilustro el perfil de un nuevo paradigma para el campo del cuidado pastoral y la disciplina de la teología pastoral con foco en la sabiduría a la luz de Dios. Luego de considerar una situación real de consejo pastoral ${ }^{4}$ haré una explicación sistemática de las dimensiones principales del nuevo modelo. He escogido el caso de una aconsejada "no-cristiana" debido a que tanto en la práctica de la capellanía en hospitales como en la de consejo, debemos aprender a atender bien a aquellas personas que no se identifican con la fe cristiana, y cuyo número va en aumento. Supongo que se nos llama a cuidar pastoralmente y también cristianamente. La naturaleza pastoral de nuestra labor está determinada, primeramente, por nuestra identidad pastoral, incluyendo ciertas

3 La noción de inteligencia moral suele utilizarse como equivalente a la de sabiduría práctica (en griego, fronesis, concepto que en la ética de Aristóteles significa la inteligencia o sabiduría de la persona buena, estrechamente asociada con la virtud y el buen carácter). Mi uso del concepto de inteligencia moral incluye una consideración comprensiva de la virtud y el carácter en términos de formación moral enraizada en la espiritualidad y definida teológicamente como tal.

4 He alterado la información que caracteriza a la ilustración que aparece más abajo con el fin de preservar la confidencialidad.

Rev. Pistis Prax., Teol. Pastor., Curitiba, v. 3, n. 2, p. 405-423, jul./dez. 2011 
formas de responsabilidad ministerial formalmente asumidas frente a la iglesia (por ejemplo mediante el licenciamiento y la acreditación de ordenación pastoral) cualquiera sea el contexto donde ejerzamos. Segundo, nuestra labor de cuidado y consejo es pastoral en la medida que trabajemos consistentemente con un marco de referencia teológico-pastoral. ${ }^{5}$ En cuanto a la expresión "cuidar cristianamente", quiero decir la calidad de cuidado espiritual que proviene de tres dimensiones interrelacionadas de la fe cristiana: una particular visión de la realidad y de la vida buena; una disposición a cuidar como una forma del amor al prójimo (y, especialmente, al "extraño") inspirada en Jesucristo; y un sentido de vocación para servir en sociedad con el Espíritu de Dios.

\section{Caso ilustrativo: Analía y su búsqueda de orientación existencial}

Analía era una mujer soltera de 37 años de edad quien recientemente había ascendido como ejecutiva de una compañía importante. Durante el año previo a la consejería se había estado preguntando si debería cancelar una amistad íntima con Mateo, su ex novio, un hombre de 39 años de edad. Mateo se había casado con otra mujer y era el padre de dos hijos, pero hacía dos años que se había separado de su esposa. De vez en cuando Analía había pensado en la posibilidad de reconectarse con Mateo, a pesar de reconocer que, aunque podían divertirse mucho juntos, en realidad eran dos personas muy diferentes e incompatibles como pareja. Varias semanas antes de comenzar la terapia Analía había tenido lo que llamó "sueños extraños" con unos ángeles que trataban de comunicarle algo. La ocasión para buscar ayuda fue su necesidad de comprender lo que estaba pasando en su vida y un deseo intenso de resolver su crisis existencial. Una persona conocida por ella y por mí le recomendó mi nombre.

Analía se había criado en una familia católica pero se consideraba persona no-religiosa y con profunda sensibilidad espiritual. De vez en

5 En el capítulo 3 de mi libro, El consejo pastoral como camino de sabiduría, presento sistemáticamente una teología pastoral del ministerio de consejo pastoral que es especialmente pertinente de cara a los desafíos y oportunidades de los contextos y las situaciones de diversidad y pluralismo. 
cuando había disfrutado la práctica de diversas expresiones de espiritualidad oriental y otras actividades tipo "nueva era" orientadas a la purificación integral, iluminación mental, aumento del aprecio de la belleza, y la libertad para crear y amar.

En la primera sesión de consejo pastoral, cuando estaba contando la historia de su vida, Analía me confió que había tenido un aborto ocho años atrás, durante el tiempo cuando estaba en pareja con Mateo. Ahora a los 37 años lamentaba profundamente esa decisión, que Mateo le había aconsejado tomar, y se sentía culpable por eso. Me indicó además que estaba segura de que la criatura hubiera sido una nena y que, de hecho, "la" había empezado a llamar Noemí (que era el nombre real de su abuela materna). Después de clarificar ciertas expectativas mutuas, acordamos reunirnos para colaborar en un proceso de consejo pastoral breve.

Analía recibió como señal esperanzadora mi observación de que el nombre "Noemî" significa "placentera" o "agradable". También advirtió que necesitaría reconsiderar recuerdos penosos y encontrar maneras de resolver constructivamente su sentido de pérdida y de culpa. La animé a que recibiera a sus sueños mentalmente con aprecio en la medida de lo posible y escribiera el contenido de aquéllos de modo que, al considerarlos juntos en las sesiones de consejo, pudiéramos encontrar significados nuevos y sentido de dirección. También acordamos que los ángeles pueden simbolizar tanto "mensajeros" como "espíritus guardianes".

Además del intento de reconciliar e integrar material inconsciente mediante el foco en sus sueños, le resultó muy útil a Analía ejercitar su imaginación en la forma de escribir cartas a "Noemí" y compartirlas conmigo, incluyendo la respuesta posible que ella podría haber recibido de su hija. Como método terapéutico, esa actividad resultó ser una manera fructífera de bregar con su aflicción y su sentido de culpa. También le ayudó a comenzar a visualizar un camino alternativo, oportunidad abierta por la experiencia única del perdón. Analía ya no estaría inhibida por el poder de un secreto sepultado y, de hecho, decidió contar lo del aborto a su madre y a su hermana, incluyendo una referencia a su labor en la terapia. Casi naturalmente, Analía comenzó a sentir la liberación de la opresión del pasado junto con un sentimiento de esperanza. 
Desde el comienzo animé a Analía a que considerara su experiencia de pérdida y aflicción no sólo como un trauma emocional sino también como una lucha espiritual. En cierto momento le confié que yo siempre oro para poder ser el mejor acompañante posible en el consejo, y que también oro a favor de las personas que aconsejo, no importa si ellas tienen o no tienen conocimiento de eso. Analía expresó que apreciaba mi oración a su favor. Es interesante apuntar finalmente que al término del proceso de terapia ella me llegó a percibir no sólo como terapeuta orientador y testigo de su proceso de sanidad en marcha, sino también como una especie de mensajero (o "angel").

El modelo de consejo pastoral que he desarrollado en torno a la sabiduría tiene cuatro componentes interrelacionados:

- una visión cuadridimensional de la realidad y el conocimiento;

- apreciación y agenda interdisciplinarias;

- metas complementarias para quienes necesitan y quien ofrece consejo pastoral;

- un propósito general junto con un enfoque fundamental.

\section{Visión cuadridimensional}

Analía había estado disfrutando la "buena vida" rodeada de muchas amistades y colegas. Era una mujer muy inteligente, llena de energía, socialmente popular, y amante de las diversiones. También había logrado mucho en el campo del trabajo. Sin embargo había comenzado a sentirse cada vez más inquieta existencialmente en su compleja relación con Mateo, su ex-pareja, y en medio de las demandas y oportunidades de su exitosa carrera profesional.

Analía estaba atravesando un nuevo capítulo en su vida, lleno de serios desafíos tales como un cambiante sentido de identidad y deseos en conflicto en las áreas de la sexualidad y la posibilidad de la maternidad, junto con un anhelo creciente de "establecerse" de alguna forma en al vida. Su malestar y desorientación incluía varios aspectos de su yo y su 
mundo. Por lo tanto, el consejo pastoral necesitaría abordar una cantidad de asuntos de naturaleza biológica, emocional, y relacional.

El modelo de consejo pastoral como práctica de sabiduría requiere sin embargo un marco de análisis y una estrategia terapéutica que van más allá de aquellas dos dimensiones -el yo y el mundo real o ambiente - aludidas en el párrafo anterior. Debemos trabajar también con las dimensiones existenciales y espirituales - lo que se ha llamado el Vacío y lo Sagrado, tal como los describió James E. Loder: "Ser humano implica el ambiente, el yo y la identidad, la posibilidad de no-ser, y la posibilidad de nuevo ser. Las cuatro dimensiones son esenciales y ninguna de ellas puede ignorarse sin que se limite decisivamente nuestra comprensión de lo que es esencialmente humano (LODER, 1989, p. 69). ${ }^{6}$ Estas cuatro dimensiones estuvieron también presentes, por cierto, en la experiencia de Analía y en su potencial de sanidad y crecimiento. Los rostros del Vacío, la amenaza implícita del no-ser la sentía con angustia existencial conectada con su profundo sentido de pérdida, especialmente la pérdida de la oportunidad de dar a luz. Su decisión de abortar, que ahora lamentaba, se acompañaba de los efectos de una aflicción suprimida acumulados por varios años. Otro rostro del Vacío para Analía era el miedo de ya no ser capaz de amar y ser amada auténticamente. Tal amenaza multidimensional debía confrontarse en la terapia al tiempo que yo la guiaría en un proceso encaminado a la experiencia de los dones de la Gracia liberadora y recreadora. El malestar de Analía podría así transformarse en una nueva experiencia de luz y amor - o sea, la negación de la negación, dialécticamente hablando - que resulta en algún grado de crecimiento hacia el "ser más" o "nuevo ser". Su sensibilidad espiritual y su búsqueda honesta de reorientación y renovación fueron desde ya recursos indispensables para la resurrección de la esperanza.

En mi práctica de consejo pastoral siempre procuro abordar las dimensiones existenciales-espirituales no importa de qué situación específica se trate. Creo que es esencial evaluar toda situación terapéutica

6 Aludimos aquí a la noción del "cuádruple evento de conocimiento" desarrollada por Loder, y que incluye el mundo vivido, el yo (self), el Vacío, y lo Sagrado. The Transforming Moment. 2nd ed. Colorado Springs: Helmers \& Howard, 1989. p. 69.

Rev. Pistis Prax., Teol. Pastor., Curitiba, v. 3, n. 2, p. 405-423, jul./dez. 2011 
cuadridimensionalmente. Es decir, no debemos sólo considerar las relaciones entre el yo y el mundo ambiental de las personas aconsejadas, lo cual generalmente determina el horizonte estrecho de otras formas de aconsejamiento y psicoterapia. Debemos también trabajar dentro del marco más amplio que incluye la amenaza del no-ser - el Vacío - y el potencial y la invitación al nuevo ser - el ámbito de lo Sagrado. Debemos además percibir y utilizar el proceso de consejo como ministerio cristiano de acuerdo a las cuatro dimensiones, por ejemplo honrando concretamente nuestra colaboración con el Espíritu de Dios en la práctica ministerial.

\section{Perspectiva y evaluación interdisciplinarias}

Analía y yo acordamos que necesitábamos abordar asuntos interrelacionados y conectados a su sentimiento de pérdida y a la culpa y la depresión resultante, sin descuidar las dinámicas sistémicas relacionadas con su trasfondo familiar y su situación interpersonal, social, y profesional presente. La agenda de consejo pastoral que identificamos incluía lo siguiente:

- La autoimagen de Analía como mujer, en relación con sus roles como profesional, amiga, y amante; junto con eso, una comprensión de la naturaleza y la dinámica de su "falso yo"7 reforzada por múltiples relaciones superficiales, aventuras sexuales, y exceso de esfuerzos profesionales;

- Relaciones con personas significativas en su vida, especialmente su familia, sus amistades, y sus ex-amantes, y el desafío de enfrentarse con la ira suprimida, sentimientos de culpa, y conflicto interior no resuelto;

7 La noción psicodinámica del "falso yo" (o falsa identidad del yo) se refiere a la interacción con el mundo social determinada mayormente por las demandas y expectativas de otras personas en vez de por las propias necesidades y deseos. El concepto ("false self") proviene de la obra de Donald W. Winnicot y su estudio de la psicodinámica del proceso de desarrollo temprano del yo. The Maturational Process and the Facilitating Environment, International University Press, Madison, 1965. 
- Identificación adecuada de necesidades y esperanzas actuales, incluyendo su sentido de dirección vocacional, y proyecciones relativas al trabajo profesional;

- Disponibilidad de recursos interiores y exteriores que podrían contribuir al proceso de sanidad.

Además de tales asuntos y otros relacionados, el enfoque de cuidado explícitamente pastoral con que trabajé motivó la identificación de varios puntos de interés para dilucidar en perspectiva teológica, tales como los siguientes:

- Aceptación de la Gracia y el cuidado en relación con las experiencias dolorosas del pasado y el abordaje inadecuado y falta de resolución de las mismas (lo cual puede caracterizarse como falla moral), junto con la posibilidad de transformación y sanidad;

- Imágenes sobre la vergüenza y la culpa, y de la aceptación, el perdón y la reconciliación, a la luz del sentido de integridad moral y responsabilidad de Analía (posiblemente conectables, de alguna manera, con la memoria reconstruida de la experiencia y la práctica de la fe cristiana en la niñez);

- El significado y la función de la espiritualidad propia de Analía y su función posible en reformar la narrativa de su historia personal, su identidad, y su sentido de vocación;

- El potencial de crecimiento espiritual como clave de plenitud humana para realizarse en forma única en una vida más sana y en la construcción de un futuro mejor.

La aplicación de evaluación y perspectivas interdisciplinarias es el segundo componente del modelo de consejo pastoral propuesto. Esta pista puede formularse como sigue: es indispensable identificar el contenido pertinente de la agenda de consejo pastoral desde una perspectiva psicológica y desde un punto de vista teológico. Por lo tanto debemos afirmar la integridad de esas disciplinas diferentes que son la psicología y la teología. Debemos evitar reducir a cualquiera de ellas en términos de la otra al tiempo que reconocemos el potencial de complementariedad que 
existe en sus respectivas contribuciones. Al mismo tiempo, debemos dar prioridad a la naturaleza pastoral y teológica de nuestra labor ministerial, incluyendo la reflexión sistemática sobre la práctica misma del consejo pastoral (HUNSINGER, 1995). ${ }^{8}$ Esa prioridad merece subrayarse debido a las maneras como la teología aborda cuestiones fundamentales sobre la vida humana y, por lo tanto, informa los aspectos normativos de nuestra labor en el consejo pastoral: ¿qué significa vivir bien a la luz del reinado de Dios y buscar sabiduría en medio de los desafíos y luchas de nuestra vida?; ¿en qué consiste la plenitud humana?; ¿cómo entendemos y promovemos la madurez, y cómo identificamos el "progreso" en el emerger humano? La teología tiene instrumentos para considerar adecuadamente tales preguntas y muchas otras, siempre en diálogo crítico y creativo con las ciencias humanas, claro está. Al ayudar a personas como Analía a tomar decisiones orientadas a una vida mejor, el consejo pastoral puede contribuir al desarrollo de la inteligencia espiritual y al crecimiento moral y espiritual, los que se pueden definir en términos de "saber vivir" y de "vida buena".

\section{Metas complementarias}

Como consejero pastoral debo proponer y reconsiderar ciertas metas que sirven no sólo para dar orientación general a mi práctica sino también para evaluar dicha práctica. Es indispensable conservar en mente tales metas para ejercer un ministerio responsable de cara a las personas aconsejadas, a mis colegas, y a la iglesia que represento y que acredita o valida mi ministerio por ordenación y mantenimiento del estatus de ministro ordenado. Algunas de las metas generales que en realidad se aplican a toda clase de situación de aconsejamiento son:

8 Véase la nota al pie número 2 con referencia a Deborah van Deusen Hunsinger y su explicación sistemática de esos tres principios epistemológicos y metodológicos en términos del "patrón de Calcedonia" aplicable a la relación ente psicología y teología. También, por la misma autora, "An Interdiciplinary Map for Christian Counselors: Theology and Psychology in Christian Counseling," en Mark R. McMinn \& Timothy R. Phillips, eds., Care for the Soul: Exploring the Intersection of Psychology and Theology. Downers Grove: InterVarsity Press, 2001. p. 218-240. 
- Recibir a los aconsejados con hospitalidad en un espacio seguro de cuidado donde pueden expresarse libremente, clarificar la naturaleza de su malestar, tomar decisiones sabias, y resultar potenciados o "empoderados" para seguir adelante.

- Representar fielmente al Cristo sanador y a la iglesia como ecología de sabiduría y plenitud humana, cuidado y sanidad, labor que incluye la posibilidad de mediar manifestaciones de Gracia con compasión y generosidad.

- Convertirme por un breve tiempo en acompañante y colaborador en el caminar hacia la (re)orientación, transformación, reconciliación, sanidad, de las personas aconsejadas.

- Ministrar como sabio consejero que practica la terapia con competencia clínica y terapéutica, especialmente al emplear los múltiples recursos que proveen la psicología y la psicoterapia como ciencia humana práctica.

Yo debía mantener en foco tales metas generales mientras trataba de ayudar a Analía a enfrentar la crisis precipitada por su actual malestar y desorientación existencial. Al mismo tiempo, sin embargo, también debía identificar los objetivos del consejo pastoral a la luz de su necesidad de apoyo y orientación. Esos objetivos específicos, que también pueden articularse como resultados deseables del proceso terapéutico, incluían los siguientes:

- Tener la experiencia de alivio emocional mediante una catarsis apropiada;

- Comprender la naturaleza de la situación de crisis por la que Analía atravesaba y apreciar e integrar la realidad de su dolor y sentido de pérdida y desorientación;

- Identificar y activar los recursos disponibles, tanto internos como externos, para manejar y eventualmente resolver saludablemente la situación de crisis

- Ayudar a fortalecer emocional y espiritualmente su sentido de identidad e integridad personal 
- Desarrollar un plan de acción realista más allá de la situación de consejo pastoral.

Yo necesitaba aplicar métodos y recursos terapéuticos comúnmente asociados a las estrategias de consejo de crisis, apoyo, y cuidado y consejo narrativo. Por lo tanto, los objetivos específicos de mis esfuerzos incluían lo siguiente:

- Dar una abierta y cuidadosa bienvenida a Analía teniendo en cuenta que ella tenía un trasfondo espiritual y teológico, y un marco referencial moral y ético, diferentes de los míos;

- Convertirme en una presencia pastoral y fuente de bienestar emocional y espiritual escuchando con sensibilidad, animándole a "decir su palabra”, y poniendo a su disposición los recursos provenientes de distintas fuentes (las ciencias humanas, la fe y la tradición cristiana y la comunidad de fe) con el fin de sostener el rescate de memorias dolorosas, la necesidad de lamentar, y el procesar el duelo;

- Ayudarle a clarificar sus propios sentimientos y a articular sus formas de comprender la experiencia de desorientación y pérdida en sus propios términos;

- Animarle a tomar nuevas decisiones saludables de cara a las nuevas realidades presentes en su vida y guiarle a poner a prueba la viabilidad del camino de restauración y sanidad por ella elegido;

- Promover el crecimiento espiritual mediante la exploración de formas de nutrir prácticas individuales y comunitarias saludables, incluyendo nuevas disciplinas tales como meditar, escribir un diario personal, y orar;

- Animar a Analía a conectarse con personas capaces de apoyarla en forma amablemente crítica y responsable más allá de nuestra relación de terapia breve;

- Comunicar mi disposición a permanecer disponible si fuera necesario reforzar el proceso de ayuda con algunas sesiones adicionales, aparte de la identificación de otros recursos de cuidado entre sus familiares y amistades. 
El tercer componente indispensable del modelo de consejo pastoral centrado en la sabiduría a la luz de Dios consiste en identificar e integrar dos clases de metas para el consejo pastoral. Por un lado, las metas (en el sentido de resultados deseados) deben seleccionarse desde la perspectiva de quienes necesitan consejo en diálogo con quien aconseja, a partir de la necesidades, esperanzas y recursos de quienes buscan ayuda. Por otro lado, quienes aconsejamos también necesitamos claridad en cuanto a las metas para la labor por cuanto estamos a cargo de guiar el proceso de aconsejemiento. Lo más importante es que el consejo pastoral cristiano procure honrar su llamado a mediar gracia y sabiduría divinas en representación de la iglesia como comunidad de cuidado y de Jesucristo como sanador, independientemente de la espiritualidad (FOWLER, 1981) $)^{9}$ de la fe (religiosa o no religiosa) de quienes reciben la ayuda. Por lo tanto, el carácter de la consejera o el consejero debe reflejar su participación continua en comunidades de fe que viven de acuerdo al evangelio del reinado de Dios en el mundo y comprometidos a ministrar no sólo como terapeutas competentes sino también como guías morales y espirituales. De hecho, tales características personales vocación, carácter, competencia, y compromiso - junto con la estructura formal de responsabilidad hacia la iglesia, son elementos esenciales del consejo verdaderamente pastoral.

\section{Propósito general}

Cada situación requiere por cierto que formulemos objetivos específicos. Cada situación también requiere que apliquemos estrategias pertinentes para alcanzar dichos objetivos. Al mismo tiempo, es obvio que todas las situaciones de consejo pastoral tienen mucho en común. Esos

9 La noción de espiritualidad en ese contexto es una construcción conceptual amplia que connota la necesidad humana fundamental de significado y valoración y la disposición a relacionarse con una poder trascendente. Siguiendo la contribución de James W. Fowler (véase, entre otros de sus escritos, Stages of Faith: Human Development and the Quest for Meaning, Harper \& Row, San Francisco, 1981), utilizo el término "fe" en sentido amplio (es decir, incluyendo también a la fe no-religiosa y humanista) con la denotación de patrones evolutivos de formas de expresar la espiritualidad a lo largo del ciclo vital humano.

Rev. Pistis Prax., Teol. Pastor., Curitiba, v. 3, n. 2, p. 405-423, jul./dez. 2011 
puntos de convergencia, centrados en términos de propósito general y proceso fundamental, apuntan a la sabiduría a la luz de Dios como la metáfora mejor para la consejería pastoral.

Analía había entrado en la relación terapéutica porque se sentía desorientada y emocionalmente desbordada. En el transcurso del proceso de terapia breve fue invitada, por cierto implícita más bien que explícitamente, a transformarse en una persona más sabia a la luz de Dios mientras trabajábamos en colaboración enfocando los desafíos y conflictos que ella estaba enfrentando en aquellos momentos de su vida. El propósito más amplio de crecer en sabiduría incluía tres aspectos inseparables para su búsqueda de alivio y resolución. Como consejero pastoral yo debía tener en mente que cada uno de tales aspectos integra dimensiones psíquicas y espirituales del ser.

\section{Crecimiento en visión}

Primero, la experiencia de consejo pastoral estaba orientada a ayudar a la aconsejada a encontrar nuevas y mejores maneras de conocer y comprender la realidad, incluyendo las dimensiones de su propia persona, el mundo social, las amenazas del Vacío, y la realidad de la gracia de lo Sagrado. Desde el punto de vista del marco normativo de la formación cristiana, Analía necesitaba crecer en cuanto a sus formas de "ver", para percibir la realidad (especialmente ella misma y otras personas) como con los ojos de Dios, metafóricamente hablando. Tal crecimiento en visión incluiría la práctica y el desarrollo de disposiciones y comportamientos identificables como una mejor capacidad para prestar atención, observar, contemplar, y admirar; para practicar el pensamiento crítico, la imaginación creadora, y el discernimiento moral y espiritual.

\section{Crecimiento en virtud}

En segundo lugar, la experiencia de consejo debía invitar a Analía a descubrir formas de ser y amar más satisfactorias, con foco particular 
en su relación con otras personas -especialmente amistades, familiares, y compañeros de trabajo - con el Espíritu de Dios, y consigo misma. En términos de formación cristiana, nos atrevemos a decir que el corazón de Analía necesitaba conformarse progresivamente al corazón de Cristo. Tal crecimiento en virtud incluiría un proceso continuo de formación y transformación, forjando los afectos y pasiones, disposiciones y actitudes los así llamados "hábitos del corazón" - y definiendo el contenido de su carácter moral y espiritual. En síntesis, durante el proceso terapéutico, de alguna manera Dios la estaba llamando a convertirse en una expresión única del amor humano capaz de reflejar el amor divino.

\section{Crecimiento en vocación}

Tercero, la experiencia de consejo pastoral procuraba capacitar a Analía para tomar buenas decisiones e invertir energías frescas en relaciones interpersonales, trabajo, descanso y juego, alimento espiritual, y servicio, y encontrar maneras de sostener tales decisiones con integridad. Necesitaba encontrar una orientación hacia la vida que fuera más libre y más esperanzada en medio de su situación social. Tal crecimiento en vocación puede considerarse teológicamente como participar en medida creciente en la vida del Espíritu en el mundo. Para Analía se podría abrir la posibilidad de una respuesta fructífera y alegre a la invitación a colaborar con Dios en actividades creativas, protectoras, liberadoras, y renovadoras. Yo esperaba que en la medida que sus maneras de ser y vivir fueran cada vez más consistentes con mi comprensión de la actividad y los propósitos de Dios, su conducta reflejaría dones tales como el sentido de la vida, amor generoso, valor, esperanza, y coraje.

El propósito general del consejo pastoral era fundamentalmente ayudar a Analía a descubrir cómo vivir una vida más íntegra, moral, y plena. Para realizar su potencial como ministerio de cuidado pastoral, el proceso terapéutico necesitaría despertar, alimentar, y capacitar su inteligencia moral y espiritual, tal como se la caracteriza más arriba. Entendida como sabiduría a la luz de Dios, la inteligencia espiritual transforma la inteligencia emocional, social, y otras formas de inteligencia toda vez que 
estas últimas tienden a promover la mera adaptación y la conformidad con la cultura dominante. En otras palabras, puede decirse que el consejo pastoral está al servicio de la transformación de la sabiduría convencional y pragmática imperante en el orden social establecido.

\section{Un enfoque fundamental}

No hay duda de que cada situación de consejo pastoral requiere que seleccionemos diversas estrategias y métodos terapéuticos apropiados. Al mismo tiempo debemos reconocer que la labor de colaboración que define a este ministerio como tal, en todos los casos debe incluir la práctica del discernimiento como aspecto esencial. Desde nuestra perspectiva pastoral y teológica percibimos que siempre debe haber una especie de "conversación” múltiple y crítica. Tal conversación incluye las historias y esperanzas de los aconsejados apreciables dentro de contextos familiares y socio-culturales específicos; las perspectivas, conocimientos e instrumentos de las ciencias humanas (especialmente los provenientes de la teoría de la personalidad, las terapias psicodinámicas, cognitivas, sistémicas, y narrativas, y las terapias sistémicas de familia); y los recursos teológicos, espirituales, y pastorales. ${ }^{10}$ Expresado en otros términos, se trata de una actividad básicamente hermenéutica que nos conduce a:

1) discernir lo que requiere una situación particular;

2) buscar alternativas y desarrollar un plan de acción;

3) a evaluar las continuas respuestas de las personas aconsejadas a los desafíos y conflictos que enfrentan.

Desde un punto de vista teológico, el contexto y el proceso del consejo pastoral incluyen más de lo que consejeros y terapeutas normalmente reconocen, al menos explícitamente. La comprensión cuadridimensional de la realidad y el conocimiento determina la naturaleza del

${ }^{10}$ La metáfora de la conversación múltiple sugiere que el consejo pastoral es una forma de hacer teología práctica que siempre debe practicarse como proceso dialéctico-hermenéutico. 
enfoque general y la actividad de discernimiento que estamos considerando. El consejo realmente pastoral ocurre no sólo en un espacio terapéutico seguro sino también en un lugar sagrado donde la presencia y actividad del Espíritu se reconoce (al menos de parte de la consejera o el consejero pastoral). Además, nos comprometemos a ministrar en colaboración con el Espíritu en el proceso de descubrir cuál es la naturaleza real de los problemas enfrentados y de las maneras mejores de abordarlos y transformarlos.

Concebida en términos bien amplios, entonces, la actividad de discernimiento condiciona significativamente el proceso (el cómo, es decir los acercamientos, métodos y técnicas) y también el contenido (el qué, o sea la agenda, los temas y asuntos a considerar) del consejo pastoral. Además, el aprendizaje de la propia práctica del discernimiento, especialmente como disciplina colaborativa, dialógica, y sostenida en oración, también se transforma en objetivo especial o resultado deseado en las personas aconsejadas en todos los casos. Por cierto, un fiel indicador de crecimiento y progreso para Analía y un sinnúmero de aconsejados es su disposición y habilidad para practicar el discernimiento. En otras palabras, crecer en sabiduría siempre incluye la capacidad para discernir y escoger sabiamente, junto con el aprendizaje para actuar y relacionarnos sabiamente con los demás de manera consistente. La sabiduría a la luz de Dios por lo tanto suple el principio guía y la metáfora central para el consejo pastoral. Y el camino de sabiduría así entendido y aplicado en la terapia y en la vida cotidiana es el proceso de conocer cómo vivir mejor en medio de nuestras circunstancias existenciales y sociales.

\section{Referências}

FOWLER, J. W. Stages of faith: human development and the quest for meaning. San Francisco: Harper \& Row, 1981.

HUNSINGER, D. van D. Theology \& pastoral counseling: a new interdisciplinary approach. Grand Rapids: Eerdmans, 1995. 
HUNSINGER, D. van D. An interdiciplinary map for christian counselors: theology and psychology in christian counseling. In: MCMINN, M. R.; PHILLIPS, T. R. (Ed.). Care for the soul: exploring the intersection of psychology and theology. Downers Grove: InterVarsity Press, 2001. p. 218-240.

LODER, J. E. The transforming moment. 2nd ed. Colorado Springs: Helmers \& Howard, 1989.

MCMINN, M. R.; PHILLIPS, T. R. (Ed.). Care for the soul: exploring the intersection of psychology and theology. Downers Grove: InterVarsity Press, 2001.

SCHIPANI, D. S. Bases eclesiológicas: la iglesia como comunidad sanadora. In: SCHIPANI, D. S.; JIMÉNEZ, P. A. (Ed.). Psicología y consejo pastoral: perspectivas hispanas. Decatur: AETH, 1997a. p. 3-26.

SCHIPANI, D. S.; JIMÉNEZ, P. A. (Ed.). Psicología y consejo pastoral: perspectivas hispanas. AETH, Decatur, 1997b.

SCHIPANI, D. S.; JIMÉNEZ, P. A. (Ed.). 0 caminho da sabedoria no aconselhamento pastoral. São Leopoldo: Sinodal, 2004.

WINNICOTT. D. The maturational process and the facilitating environment. Madison: International University Press, 1965.

Recebido: 01/12/2010

Recieved: 12/01/2010

Aprovado: 22/12/2010

Approved: 12/22/2010 